\title{
REMARKS ON THE FLUX GROUPS
}

\author{
JAROSLAW KȨDRA
}

\begin{abstract}
Under some topological assumptions, I give a new estimation of the rank of flux groups and provide a method of constructions of symplectic aspherical manifolds.
\end{abstract}

\section{Introduction}

Let $(M, \omega)$ be a compact symplectic manifold and $\operatorname{Symp}(M, \omega)$ denotes the group of symplectomorphisms of $(M, \omega)$. Define the flux homomorphism

$$
\begin{gathered}
F: \pi_{1}\left(\operatorname{Symp}_{0}(M, \omega)\right) \rightarrow H^{1}(M ; \mathbb{R}) \\
F(\xi)[a]=\int_{\xi_{t} a} \omega,
\end{gathered}
$$

where $\xi_{t} a$ denotes the trace of a loop $a$ under the isotopy $\left\{\xi_{t}\right\}$ representing $\xi$. By definition the flux group $\Gamma_{\omega}$ is the image of the flux homomorphism.

In this paper, we investigate properties the flux groups. Lalonde, McDuff and Polterovich proved that the rank over $\mathbb{Z}$ of a flux group of $(M, \omega)$ is not greater than $b_{1}(M)$ [LMP2, Theorem 2.D]. We extend this result as it is stated in Theorem A. The proofs of our results (except of the second assertion of Theorem A) are mainly based on the work of Gottlieb [Got]. Similarly, Lupton and Oprea used the Gottlieb theory to investigation of properties of circle actions on, so called, cohomologicaly symplectic manifolds [LO].

Let's briefly look at the contents of the present work.

Theorem A. Let $(M, \omega)$ be a compact symplectic manifold satisfying one of the following conditions:

1. some Chern number of $(M, \omega)$ is nonzero

2. $M$ is aspherical and $Z\left(\pi_{1}(M)\right) \subset\left[\pi_{1}(M), \pi_{1}(M)\right]$

3. $\operatorname{ker} A \subset\left[\pi_{1}(M), \pi_{1}(M)\right]+$ torsion, where $A: \pi_{1}(M) \rightarrow \operatorname{Aut}\left(\pi_{*}(M)\right)$ is the action of the fundamental group on homotopy groups.

Then $\Gamma_{\omega} \subset \operatorname{ker}\left[\cup[\omega]^{n-1}: H^{1}(M ; \mathbb{R}) \rightarrow H^{2 n-1}(M ; \mathbb{R})\right]$ and the rank of $\Gamma_{\omega}$ over $\mathbb{Z}$ is not greater than $\operatorname{dim} \operatorname{ker}\left(\cup[\omega]^{n-1}\right)$.

Received November 30, 1999.

Research supported by the KBN under grants numbers 2 PO3A 02015 and 2 PO3A 02314. 
We prove this theorem using various methods. The case (1) follows from the properties of the Wang sequence associated to a relevant fibration (cf. [LMP2]), whereas the cases (2) and (3) uses the Gottlieb theory. The proof of the statement about the rank of flux groups is a variant of the argument introduced by Lalonde, McDuff and Polterovich in [LMP2, Theorem 2D].

The above theorem provides an immediate corollary for Lefschetz manifolds. Recall that a compact symplectic manifold $(M, \omega)$ is called Lefschetz if the multiplication $\cup[\omega]^{n-1}: H^{1}(M ; \mathbb{R}) \rightarrow H^{2 n-1}(M ; \mathbb{R})$ is an isomorphism. Then we have

Corollary A. Let $(M, \omega)$ satisfies the hypothesis of Theorem A. If in addition it is Lefschetz then its flux group is trivial.

The next theorem is concerned with aspherical manifolds and develops the second part of Theorem A.

Theorem B. Let $(M, \omega)$ be a compact aspherical symplectic manifold. If either Euler characteristic of $M$ is nonzero or $\pi_{1}(M)$ has trivial center then its flux group $\Gamma_{\omega}$ is trivial.

Notice that the above theorem does not depend on the choice of symplectictic form. The reason is probably that in this case the groups of symplectomorphisms are 1-connected or even contractible.

The second section is devoted to construction of aspherical symplectic manifolds. We use construction of symplectic sums due to Gompf [Gom] (see also $[\mathrm{MW}]$ ) and give conditions under which the resulting manifold is aspherical. At the end, we apply this construction to give an example of an aspherical symplectic manifold with zero Chern numbers and trivial flux group.

\section{The proofs}

Given an element $\xi \in \pi_{1}\left(\operatorname{Symp}_{0}(M, \omega)\right)$ there is associated (up to isomorphism) a symplectic fibration over two sphere $(M, \omega) \rightarrow P_{\xi} \rightarrow S^{2}$ as follows [LMP2].

$$
P_{\xi}=M \times D^{2} \cup_{\xi_{t}} M \times D^{2},
$$

where $D^{2}$ denotes unit disc and $\left(x, e^{2 \pi i t}\right) \cong\left(\xi_{t}(x), e^{-2 \pi i t}\right)$. Fibrations over $S^{2}$ give raise to the following exact sequences called the Wang sequences:

$$
\begin{gathered}
\ldots \rightarrow H_{k+2}\left(P_{\xi}\right) \rightarrow H_{k}(M) \stackrel{\partial_{\xi}}{\longrightarrow} H_{k+1}(M) \stackrel{i_{*}}{\longrightarrow} H_{k+1}\left(P_{\xi}\right) \rightarrow \ldots \\
\ldots \rightarrow H^{k}\left(P_{\xi}\right) \stackrel{i^{*}}{\longrightarrow} H_{k}(M) \stackrel{\partial_{\xi}^{*}}{\longrightarrow} H_{k-1}(M) \rightarrow H_{k+1}\left(P_{\xi}\right) \rightarrow \ldots,
\end{gathered}
$$

where $\partial_{\xi}[a]=\left[\xi_{t} a\right]$ and $\left\langle\partial_{\xi}^{*} \alpha,[a]\right\rangle=\left\langle\alpha, \partial_{\xi}[a]\right\rangle$. Here $\langle$,$\rangle denotes the usual$ pairing between homology and cohomology. Notice that with the above notation we have that the flux satisfies the following

$$
F(\xi)=\partial_{\xi}^{*}[\omega] .
$$


The crucial property of Wang homomorphism $\partial_{\xi}^{*}$ is that it is a derivation [Sp], i.e.,

$$
\partial_{\xi}^{*}(\alpha \cup \beta)=\partial_{\xi}^{*}(\alpha) \cup \beta+(-1)^{\operatorname{deg}(\alpha)} \alpha \cup \partial_{\xi}^{*}(\beta) .
$$

Lemma 2.1 ([M1]). Let $c_{i}(M, \omega)$ denotes $i^{\text {th }}$ Chern class of $M$ with respect to any almost complex structure compatible with $\omega$. Then

$$
\partial_{\xi}^{*}\left(c_{i}(M, \omega)\right)=0
$$

for $\xi \in \pi_{1}\left(\operatorname{Symp}_{0}(M, \omega)\right)$.

Proof. Let $(M, \omega) \rightarrow P_{\xi} \rightarrow S^{2}$ be the symplectic fibration corresponding to $\xi$ and consider the subbundle $V$ ert $\subset T P_{\xi}$ consisting of vectors tangent to the fibers. Since the fibration $P_{\xi}$ is symplectic then $V$ ert is symplectic vector bundle which Chern classes restrict to the Chern classes of $(M, \omega)$ :

$$
i^{*} c_{i}(\text { Vert })=c_{i}\left(i^{*} \text { Vert }\right)=c_{i}(T M)=c_{i}(M) .
$$

Then it follows from the exactness of Wang sequence that $\partial_{\xi}^{*}\left(c_{i}(M, \omega)\right)=0$.

Let $C(M)$ denotes the space of continuous maps from $M$ to $M$ with the open compact topology. We define an evaluation $e v_{c}: C(M) \rightarrow M$ by $e v_{c}(f)=f\left(x_{0}\right)$, $x_{0} \in M$. Similarly $e v_{s}$ is the evaluation defined on $\operatorname{Symp}(M, \omega)$. We will use the same notation for the maps induced on the fundamental groups. By $\widetilde{e v_{s}}$ we denote a homomorphism from $\pi_{1}\left(\operatorname{Symp}_{0}(M, \omega)\right)$ to $H_{1}(M)=H_{1}(M ; \mathbb{Z}) /$ torsion which is composition of $e v_{s}$ with the obvious maps.

Lemma 2.2 ([LMP1]). The following diagram is commutative up to positive constant

$$
\begin{gathered}
\pi_{1}\left(\operatorname{Symp}_{0}(M, \omega)\right) \stackrel{\sqrt[\overline{e v}]{\longrightarrow}}{\longrightarrow} H_{1}(M) \stackrel{P D}{\longrightarrow} H^{2 n-1}(M) \\
\downarrow \\
\pi_{1}\left(\operatorname{Symp}_{0}(M, \omega)\right) \stackrel{F}{\longrightarrow} H^{1}(M ; \mathbb{R}) \stackrel{\cup[\omega]^{n-1}}{\longrightarrow} H^{2 n-1}(M ; \mathbb{R}) .
\end{gathered}
$$

Remark 2.3. It follows from the above diagram that the flux conjecture holds for Lefschetz manifolds. Indeed, the image of the up arrows is discrete in $H^{2 n-1}(M ; \mathbb{R})$ and since $\cup[\omega]^{n-1}$ is an isomorphism then $\Gamma_{\omega}$ is also discrete.

Proof of Theorem A. First we prove that $\Gamma_{\omega} \subset \operatorname{ker} \cup[\omega]^{n-1}$.

(1) Suppose that some Chern number of $(M, \omega)$ is nonzero. It means that the product of some Chern classes, say $c_{k_{1}} \cup \ldots \cup c_{k_{m}} \neq 0$ in the top cohomology. It means that $c_{k_{1}} \cup \ldots \cup c_{k_{m}}=\lambda[\omega]^{n}$, for a nonzero $\lambda \in \mathbb{R}$. According to the Lemma 2.1 we obtain that

$$
0=\partial_{\xi}^{*}\left(c_{k_{1}} \cup \ldots \cup c_{k_{m}}\right)=\partial_{\xi}^{*}\left(\lambda[\omega]^{n}\right)=\lambda n \partial_{\xi}^{*}[\omega] \cup[\omega]^{n-1}=\lambda n F(\xi) \cup[\omega]^{n-1},
$$

which finishes the first part. 
(2) Now let $M$ be aspherical and $Z\left(\pi_{1}(M)\right) \subset\left[\pi_{1}(M), \pi_{1}(M)\right]$. Denote by $C_{0}(M)$ the identity component of $C(M)$. Let's consider the following diagram

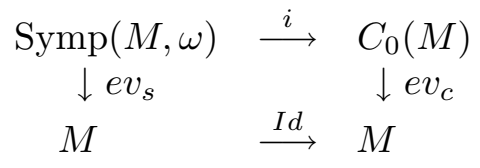

where the vertical arrows are the evaluations. Gottlieb showed in [Go, Theorem III.2] that

$$
e v_{c}: \pi_{1}\left(C_{0}(M)\right) \stackrel{\cong}{\longrightarrow} Z\left(\pi_{1}(M)\right),
$$

so passing to the maps on the fundamental groups induced by the above diagram, we get that

$$
e v_{s}(\xi)=0
$$

for every $\xi \in \pi_{1}\left(\operatorname{Symp}_{0}(M, \omega)\right)$. Thus, due to Lemma 2.2, $F(\xi) \cup[\omega]^{n-1}=0$, which finishes the second part.

(3) The proof of this point is the same as previous one since, once more due to Gottlieb [Go, Theorem I.4], we have that $e v_{c}\left(\pi_{1}\left(C_{0}(M)\right)\right) \subset \operatorname{ker} A$.

Now we have proved that $\Gamma_{\omega} \subset \operatorname{ker} \cup[\omega]^{n-1}$. For the proof of the second assertion of Theorem A we need the following lemma (see [LMP2, Theorem 2.A]).

Lemma 2.4. Let $\omega$ and $\omega^{\prime}$ be two symplectic forms on $M$. If $\xi \in \pi_{1}(\operatorname{Diff}(M))$ can be represented by a loops in $\operatorname{Symp}(M, \omega)$ and $\operatorname{Symp}_{0}\left(M, \omega^{\prime}\right)$ then $\partial_{\xi}^{*}[\omega]=0$ iff $\partial_{\xi}^{*}\left[\omega^{\prime}\right]=0$.

We have to show that the rank over $\mathbb{Z}$ of flux group $\Gamma_{\omega}$ is not greater than dimension of ker $\cup\left[\omega^{n-1}\right]$. The proof is in the same vein as the proof of Theorem 2.D in [LMP2].

Let $\omega_{\epsilon}$ denotes a rational symplectic form which is a small perturbation of $\omega$. Then

$$
\operatorname{dim} \operatorname{ker} \cup\left[\omega^{n-1}\right] \geq \operatorname{dim} \operatorname{ker} \cup\left[\omega_{\epsilon}^{n-1}\right]
$$

and $c_{i}\left(M, \omega_{\epsilon}\right)=c_{i}(M, \omega)$. Thus $\left(M, \omega_{\epsilon}\right)$ satisfies assumptions of Theorem A.

Suppose that there exist elements $\xi_{1}, \ldots, \xi_{k} \in \pi_{1}\left(\operatorname{Symp}_{0}(M, \omega)\right)$ such that $F\left(\xi_{1}\right), \ldots, F\left(\xi_{k}\right) \in \Gamma_{\omega}$ are linearly independent over $\mathbb{Z}$ and $k>\operatorname{dim} \operatorname{ker} \cup\left[\omega^{n-1}\right]$. Let $\xi_{1}^{\epsilon}, \ldots, \xi_{k}^{\epsilon} \in \pi_{1}\left(\operatorname{Symp}\left(M, \omega_{\epsilon}\right)\right.$ are represented by perturbations of loops representing $\xi_{1}, \ldots, \xi_{k} \in \pi_{1}\left(\operatorname{Symp}_{0}(M, \omega)\right)$. Then the fluxes $F_{\epsilon}\left(\xi_{j}^{\epsilon}\right)$ are rational classes and are contained in ker $\cup\left[\omega_{\epsilon}^{n-1}\right]$, according to already proved part of Theorem A. It follows that some of their nontrivial combination over $\mathbb{Z}$ equals zero: $\sum_{j} m_{j} F_{\epsilon}\left(\xi_{j}^{\epsilon}\right)=0, m_{j} \in \mathbb{Z}, j=1, \ldots, k$. Due to Lemma 2.4, we get that $\sum_{j} m_{j} F\left(\xi_{j}\right)=0$ which gives the contradiction and completes the proof.

Proof of Theorem B. Here we again use the Gottlieb theory, namely the fact that for a manifold $M$ (not symplectic in general) satisfying the hypothesis of the theorem the space $C_{0}(M)$ is contractible. Now suppose that there exists $\xi \in \pi_{1}\left(\operatorname{Symp}_{0}(M, \omega)\right)$ with $F(\xi) \neq 0$. It means that $\partial_{\xi}^{*}[\omega] \neq 0$, but Wang sequence depends only on the homotopy type of $P_{\xi}$ and the latter is homotopy 
equivalent to the trivial fibration, due to contractibility of $C_{0}(M)$. Thus we cannot have $\partial_{\xi}^{*}[\omega] \neq 0$.

\section{Symplectic aspherical surgery}

The asphericity of manifolds is a common assumption it the present paper, so that we shall give a method of construction of aspherical symplectic manifolds. In fact, the construction is due to Gompf [Gom, MW] and we only give conditions which ensure the asphericity of the resulting manifold.

Let $\left(M_{1}, \omega_{1}\right),\left(M_{2}, \omega_{2}\right)$ and $(S, \omega)$ be symplectic manifolds, where $S$ is closed and $\operatorname{dim} M_{1}=\operatorname{dim} M_{2}=\operatorname{dim} S-2$. Let $f_{i}: S \rightarrow M_{i}$ be symplectic embeddings with normal bundles $N_{i}, i=1,2$. Suppose that $c_{1}\left(N_{1}\right)=-c_{1}\left(N_{2}\right)$. Then let $M_{1} \sharp_{S} M_{2}$ denotes the Gompf symplectic sum of $\left(M_{1}, \omega_{1}\right)$ and $\left(M_{2}, \omega_{2}\right)$ [Gom].

Proposition 3.1 (aspherical surgery). If in the above situation

1. $S$ and $M_{i}-f_{i}(S)$ are aspherical, $i=1,2$,

2. the embeddings $f_{i}$ induce monomorphisms $\pi_{1}\left(\partial N_{i}\right) \rightarrow \pi_{1}\left(M_{i}-N_{i}\right)=\Gamma_{i}$, then the symplectic sum $M_{1} \sharp_{S} M_{2}$ is aspherical.

Proof. The idea of the proof is to show that the universal covering of $M_{1} \sharp_{S} M_{2}$ is homotopy equivalent to a tree. Notice that this fact is also proved in [Br,proof of Theorem 7.3] by showing that the higher homotopy groups vanish.

It follows from the second assumption and van Kampen's theorem that the fundamental group $\Gamma$ of $M_{1} \sharp_{S} M_{2}$ is the following amalgamated product $\Gamma_{1} *_{\Gamma_{0}} \Gamma_{2}$, where $\Gamma_{0}=\pi_{1}\left(N_{0}\right)$ and $N_{0}$ is $N_{1}$ without a the zero section. Thus there exists a tree $T$ on which $\Gamma$ acts freely and the fundamental domain of this action is the edge of $T$ called the graph of groups [Se, Theorem 7]. It follows from the construction of $T$ that there exists a map $h$ from the universal covering of $M_{1} \sharp_{S} M_{2}$ to that tree such that preimage of an egdge is homotopy equivalent to $\tilde{N}_{0}$ and preimage of a vertex is $M_{i}-f_{i}(S)$ for $i=1$ or 2 . Here the tilde denotes universal covering. Since these preimages are contractible, due to asphericity, then $h$ is a homotopy equivalence which ensures the contractibility of $\widetilde{M_{1} \sharp_{S} M_{2}}$. The latter means that $M_{1} \sharp_{S} M_{2}$ is aspherical.

The first part of Theorem A may suggest that the flux groups are nontrivial if the Chern numbers are zero. In the following example we construct an aspherical symplectic manifold with zero Chern numbers and trivial flux group.

Example. Let $M_{1}=T_{\psi}^{2}$ be the mapping torus, where $\psi: T^{2} \rightarrow T^{2}$ is defined by the following matrix

$$
\left[\begin{array}{ll}
2 & 1 \\
1 & 1
\end{array}\right]
$$

In other words, $M_{1}=\mathbb{R}^{2} \times T^{2} / \sim$, where $(a+1, b, x, y) \sim(a, b, x, y)$ and $(a, b+$ $1, x, y) \sim(a, b, 2 x+y, x+y)$. We endow $M_{1}$ with the invariant symplectic structure $\omega_{1}$ coming from the standard symplectic structure on $\mathbb{R}^{4}$. This ensures that Chern classes of $\left(M_{1}, \omega_{1}\right)$ are zero. Moreover, $M_{1}$ is also the total space of 
symplectic $T^{2}$-bundle over $T^{2}$, which means that we can symplectically embed a torus as the fibre. Let $M_{2}=\left(T_{\varphi}^{2}, \omega_{2}\right)$ be another mapping torus with $T^{2}$ embedded as a section or a fibre (in both cases its normal bundle is trivial). Now we perform a symplectic normal connected sum $M_{1} \sharp_{T^{2}} M_{2}$ [Gom, MW].

Let's now compute fundamental groups that we need.

$$
\pi_{1}\left(M_{1}-N\right)=(\mathbb{Z} * \mathbb{Z}) \times_{\psi} \mathbb{Z}^{2}
$$

where $\psi: \mathbb{Z} * \mathbb{Z} \rightarrow \operatorname{AUT}\left(\mathbb{Z}^{2}\right)$ is defined by $\psi(a)=\operatorname{Id}$ and $\psi(b)=\left[\begin{array}{ll}2 & 1 \\ 1 & 1\end{array}\right]$, where $a, b$ are the generators and $N$ denotes the normal bundle of the fibre. It is easy to see that the center of the above group is trivial. The boundary of the normal bundle to the fibre is three dimensional torus and its inclusion to $M_{1}-N$ induces the following monomorphism of the fundamental groups

$$
(k, l, m) \mapsto\left(\left(a b a^{-1} b^{-1}\right)^{k}, l, m\right) .
$$

According to computations of Chern numbers of symplectic sum in [MW], we get that the Chern numbers of $M_{1} \sharp_{T^{2}} M_{2}$ are zero because Chern classes of $\left(M_{1}, \omega_{1}\right)$ and $\left(M_{2}, \omega_{2}\right)$ are zero and Euler characteristic of $T^{2}$ is zero as well. Since the center of an amalgamated $G *_{K} H$ product is equal to $i^{-1}(Z(G)) \cap$ $j^{-1}(Z(H))$, where $i: K \rightarrow G, j: K \rightarrow H$ are inclusions, then we obtain that $\pi_{1}\left(M_{1} \sharp_{T^{2}} M_{2}\right)$ has trivial center. Due to Theorem B, the flux group of $M_{1} \sharp_{T^{2}} M_{2}$ is trivial.

Note that the above example is universal in the sense that a symplectic manifold which is a result of the Gompf sum with the manifold $M_{1}$ has always trivial center, thus in the aspherical case its flux is always trivial.

\section{Acknowledgements}

I am grateful to Dusa McDuff for suggesting me the proof of Theorem A(1), which I previously proved using real homotopy theory $[\mathrm{K}]$ and to Tadek Januszkiewicz for drawing my attention to the "Trees" [Se].

Also, I acknowledge the support of the Foundation for Polish Science (FNP).

\section{References}

[B] K.S. Brown, Cohomology of Groups, Graduate Texts in Mathematics, 87, SpringerVerlag, New York-Berlin, 1982.

[Gom] R. Gompf, A new construction of symplectic manifolds, Ann. of Math. 142 (1995), 527-595.

[Got] D. Gottlieb, A certain subgroup of the fundamental group, Amer. J. Math. 87 (1965), 840-856.

[K] J. Kȩdra, KS-models and loops in the group of symplectomorphisms, preprint.

[LMP1] F. Lalonde, D. McDuff, and L. Polterovich, On the flux conjectures, CRM Proceedings and Lecture Notes, 15, Amer. Math. Soc., Providence, RI, 1998, pp. 69-85.

[LMP2] _ Topological rigidity of Hamiltonian loops and quantum cohomology, Invent. Math. 135 (1999), 369-385.

[LO] G. Lupton and J. Oprea, Cohomologically symplectic spaces: toral actions and the Gottlieb group, Trans. Amer. Math. Soc. 347 (1995), 261-288. 
[MW] J. McCarthy and J. Wolfson, Symplectic normal connect sum, Topology 33 (1994), 729-764.

[M1] D. McDuff, Symplectic diffeomorphisms and the flux homomorphism, Invent. Math. 77 (1984), 353-366.

[M2] , Fibrations in symplectic topology, Proceedings of the ICM in Berlin, Doc. Math. 1998, Extra Vol. I, 339-357.

[Se] J.-P. Serre, Trees, Springer-Verlag, Berlin-New York, 1980.

[Sp] E. Spanier, Algebraic topology, McGraw-Hill Book Co., New York-Toronto, Ont.London, 1966.

Instytut Matematyczny U. Wr., Pl.Grunwaldzki 2/4, 50-384 Wroclaw, POLAND

Instytut Matematyki U.S., Ul.Wielkopolska 15, 70-467 Szczecin, POLAND

E-mail address: jkedr@math.uni.wroc.pl 